\title{
Power subgroups of the extended Hecke groups
}

Zehra Sarigedik, Sebahattin Ikikardes, and Recep Sahin 


\title{
POWER SUBGROUPS OF THE EXTENDED HECKE GROUPS
}

\author{
ZEHRA SARIGEDIK, SEBAHATTIN İKIKARDES, AND RECEP SAHIN
}

Received 22 April, 2014

\begin{abstract}
We consider the extended Hecke groups $\bar{H}\left(\lambda_{q}\right)$ generated by $T(z)=-1 / z, S(z)=$ $-1 /\left(z+\lambda_{q}\right)$ and $R(z)=1 / \bar{z}$ with $\lambda_{q}=2 \cos (\pi / q)$ for $q \geq 3$ integer. In this article, we study the abstract group structures of the power subgroups $\bar{H}^{m}\left(\lambda_{q}\right)$ of $\bar{H}\left(\lambda_{q}\right)$ for each positive integer $m$. Then, we give the relations between commutator subgroups and power subgroups.
\end{abstract}

2010 Mathematics Subject Classification: 20H10; 11F06

Keywords: extended Hecke groups, power subgroups

\section{INTRODUCTION}

In [4], Erich Hecke introduced the groups $H(\lambda)$ generated by two linear fractional transformations

$$
T(z)=-\frac{1}{z} \text { and } S(z)=-\frac{1}{z+\lambda},
$$

where $\lambda$ is a fixed positive real number.

E. Hecke showed that $H(\lambda)$ is discrete if and only if $\lambda=\lambda_{q}=2 \cos \frac{\pi}{q}, q \geq 3$ integer, or $\lambda \geq 2$. We will focus on the discrete case with $\lambda<2$, i.e., those with $\lambda=\lambda_{q}, q$ an integer $\geq 3$. These groups have come to be known as the Hecke Groups, and we will denote them $H\left(\lambda_{q}\right)$ for $q \geq 3$. The Hecke group $H\left(\lambda_{q}\right)$ is isomorphic to the free product of two finite cyclic groups of orders 2 and $q$ and it has a presentation

$$
H\left(\lambda_{q}\right)=<T, S \mid T^{2}=S^{q}=I>\cong C_{2} * C_{q} .
$$

Also $H\left(\lambda_{q}\right)$ has the signature $(0 ; 2, q, \infty)$, that is, all the groups $H\left(\lambda_{q}\right)$ are triangle groups. The first several of these groups are $H\left(\lambda_{3}\right)=\Gamma=P S L(2, \mathbb{Z})$ (the modular group), $H\left(\lambda_{4}\right)=H(\sqrt{2}), H\left(\lambda_{5}\right)=H\left(\frac{1+\sqrt{5}}{2}\right)$, and $H\left(\lambda_{6}\right)=H(\sqrt{3})$. It is clear that $H\left(\lambda_{q}\right) \subset P S L\left(2, \mathbb{Z}\left[\lambda_{q}\right]\right)$, for $q \geq 4$. The groups $H(\sqrt{2})$ and $H(\sqrt{3})$ are of particular interest, since they are the only Hecke groups, aside from the modular group, whose elements are completely known.

The extended Hecke group, denoted by $\bar{H}\left(\lambda_{q}\right)$, has been defined by adding the reflection $R(z)=1 / \bar{z}$ to the generators of the Hecke group $H\left(\lambda_{q}\right)$, for $q \geq 3$ integer, 
in $[9,10]$ and [6]. Thus, the extended Hecke group $\bar{H}\left(\lambda_{q}\right)$ has a presentation,

$\bar{H}\left(\lambda_{q}\right)=<T, S, R \mid T^{2}=S^{q}=R^{2}=I, R T=T R, R S=S^{q-1} R>\cong D_{2} * C_{2} D_{q}$.

The Hecke group $H\left(\lambda_{q}\right)$ is a subgroup of index 2 in $\bar{H}\left(\lambda_{q}\right)$.

Now we give some information about the power subgroups of $\bar{H}\left(\lambda_{q}\right)$.

Let $m$ be a positive integer. Let us define $\bar{H}^{m}\left(\lambda_{q}\right)$ to be the subgroup generated by the $m^{t h}$ powers of all elements of $\bar{H}\left(\lambda_{q}\right)$. The subgroup $\bar{H}^{m}\left(\lambda_{q}\right)$ is called the $m-t h$ power subgroup of $\bar{H}\left(\lambda_{q}\right)$. As fully invariant subgroups, they are normal in $\bar{H}\left(\lambda_{q}\right)$.

From the definition one can easily deduce that

$$
\bar{H}^{m k}\left(\lambda_{q}\right) \leq \bar{H}^{m}\left(\lambda_{q}\right)
$$

and

$$
\bar{H}^{m k}\left(\lambda_{q}\right) \leq\left(\bar{H}^{m}\left(\lambda_{q}\right)\right)^{k} .
$$

Using the last two inequalities imply that $\bar{H}^{m}\left(\lambda_{q}\right) \cdot \bar{H}^{k}\left(\lambda_{q}\right)=\bar{H}^{(m, k)}\left(\lambda_{q}\right)$ where $(m, k)$ denotes the greatest common diviser of $m$ and $k$.

The power subgroups of the Hecke groups $H\left(\lambda_{q}\right)$ have been studied and classified in [2,3] and [5]. For $q \geq 3$ prime, the power subgroups of the extended Hecke groups $\bar{H}\left(\lambda_{q}\right)$ were studied by Sahin, Ikikardes and Koruoğlu in [11,12] and [13].

The aim of this paper is to study the power subgroups $\bar{H}^{m}\left(\lambda_{q}\right)$ of the extended Hecke groups $\bar{H}\left(\lambda_{q}\right), q \geq 3$ integer. For each positive integer $m$, we determine the abstract group structures and generators of $\bar{H}^{m}\left(\lambda_{q}\right)$. Also, we give the signatures of $\bar{H}^{m}\left(\lambda_{q}\right)$. To get all these results, we use the techniques of combinatorial group theory (Reidemeister-Schreier method, permutation method and Riemann-Hurwitz formula). Finally, we give the relations between commutator subgroups and power subgroups.

\section{The GROUP STRUCTURE OF POWER SUbGRoups OF $\bar{H}\left(\lambda_{q}\right)$}

Now we consider the presentation of the extended Hecke group $\bar{H}\left(\lambda_{q}\right)$ given in (1.1):

$$
\bar{H}\left(\lambda_{q}\right)=<T, S, R \mid T^{2}=S^{q}=R^{2}=I, T R=R T, R S=S^{-1} R>
$$

Firstly, we find a presentation for the quotient $\bar{H}\left(\lambda_{q}\right) / \bar{H}^{m}\left(\lambda_{q}\right)$ by adding the relation $X^{m}=I$ for all $X \in \bar{H}\left(\lambda_{q}\right)$ to the presentation of $\bar{H}\left(\lambda_{q}\right)$. The order of $\bar{H}\left(\lambda_{q}\right) / \bar{H}^{m}\left(\lambda_{q}\right)$ gives us the index. We have,

$$
\begin{gathered}
\bar{H}\left(\lambda_{q}\right) / \bar{H}^{m}\left(\lambda_{q}\right)<T, S, R \mid T^{2}=S^{q}=R^{2}=(T R)^{2}=(R S)^{2}=T^{m}=S^{m} \\
=R^{m}=(T S)^{m}=(R S)^{m}=(T R)^{m}=\ldots=I>.
\end{gathered}
$$


Thus we use the Reidemeister-Schreier process to find the generators and the presentations of the power subgroups $\bar{H}^{m}\left(\lambda_{q}\right), q \geq 3$ integer (for the method, please see [2] and [5]).

Firstly, we now discuss the group theoretical structure of these subgroups for $q \geq 3$ odd integer. We start with the case $m=2$.

Theorem 1. 1) If $q \geq 3$ is an odd integer, then $\bar{H}^{2}\left(\lambda_{q}\right)$ is the free product of two finite cyclic groups of order q, i.e.,

$$
\bar{H}^{2}\left(\lambda_{q}\right)=\left\langle S, T S T \mid S^{q}=(T S T)^{q}=I\right\rangle \cong C_{q} * C_{q} .
$$

2) If $q>3$ is an even integer, then $\bar{H}^{2}\left(\lambda_{q}\right)$ is the free product of the infinite cyclic group and two finite cyclic groups of order $q / 2$, i.e.,

$$
\begin{aligned}
\bar{H}^{2}\left(\lambda_{q}\right) & =\left\langle S^{2}, T S^{2} T, T S T S^{-1} \mid\left(S^{2}\right)^{q / 2}=\left(T S^{2} T\right)^{q / 2}=\left(T S T S^{-1}\right)^{\infty}=I\right\rangle, \\
& \cong C_{q / 2} * C_{q / 2} * \mathbb{Z} .
\end{aligned}
$$

Proof. 1) By (2.1), we have

$$
\bar{H}\left(\lambda_{q}\right) / \bar{H}^{2}\left(\lambda_{q}\right) \cong\left\langle T, R \mid T^{2}=R^{2}=(T R)^{2}=I\right\rangle \cong C_{2} \times C_{2},
$$

since $S^{2}=S^{q}=I$ and $(2, q)=1$. Now we can choose $\{I, T, R, T R\}$ as a Schreier transversal for $\bar{H}^{2}\left(\lambda_{q}\right)$. According to the Reidemeister-Schreier method (see [8]), we get the generators of $\bar{H}^{2}\left(\lambda_{q}\right)$ as the followings :

$$
\begin{aligned}
& \text { I.T. }(T)^{-1}=I, \quad \text { I.S. }(I)^{-1}=S, \quad \text { I.R. }(R)^{-1}=I \text {, } \\
& \begin{array}{lll}
\text { T.T. }(I)^{-1}=I, & \text { T.S. }(T)^{-1}=T S T^{-1}, & \text { T.R. }(T R)^{-1}=I,
\end{array} \\
& \text { R.T. }(T R)^{-1}=R T R T, \quad R . S .(R)^{-1}=R S R^{-1}, \quad \text { R.R. }(I)^{-1}=I \text {, } \\
& \text { TR.T. }(R)^{-1}=T R T R, \quad T R . S .(T R)^{-1}=T R S R^{-1} T^{-1}, \quad T R . R .(T)^{-1}=I .
\end{aligned}
$$

Since $T R T R=R T R T=I, R S R^{-1}=S^{-1}$ and $T R S R^{-1} T^{-1}=(T S T)^{-1}$, the generators of $\bar{H}^{2}\left(\lambda_{q}\right)$ are $S$ and $T S T$. Thus $\bar{H}^{2}\left(\lambda_{q}\right)$ has a presentation

$$
\bar{H}^{2}\left(\lambda_{q}\right)=\left\langle S, T S T \mid S^{q}=(T S T)^{q}=I\right\rangle \cong C_{q} * C_{q} .
$$

Also, using the permutation method (see [14]) and the Riemann-Hurwitz formula, we get the signature of $\bar{H}^{2}\left(\lambda_{q}\right)$ as $(0 ; q, q, \infty)=\left(0 ; q^{(2)}, \infty\right)$.

2) By 2.1 , the quotient group $\bar{H}\left(\lambda_{q}\right) / \bar{H}^{2}\left(\lambda_{q}\right)$ is

$$
\begin{aligned}
\bar{H}\left(\lambda_{q}\right) / \bar{H}^{2}\left(\lambda_{q}\right) & \cong\left\langle T, S, R \mid T^{2}=S^{2}=R^{2}=(T R)^{2}=(R S)^{2}=(T S)^{2}=I\right\rangle \\
& \cong C_{2} \times C_{2} \times C_{2},
\end{aligned}
$$


since $S^{2}=S^{q}=I$. Now we can choose Schreier transversal as $\{I, T, S, R, T S, T R$, $S R, T S R\}$. According to the Reidemeister-Schreier method, all possible products are

$$
\begin{aligned}
& \text { I.T. }(T)^{-1}=I, \quad \text { TS.T. }(S)^{-1}=\text { TSTS }^{-1}, \\
& \text { T.T. }(I)^{-1}=I, \quad \text { TR.T. }(R)^{-1}=I, \\
& \text { S.T. }(T S)^{-1}=S T S^{-1} T, \quad S R . T .(T S R)^{-1}=S R T R S^{-1} T, \\
& \text { R.T. }(T R)^{-1}=I, \quad \text { TSR.T. }(S R)^{-1}=T_{S R T R S^{-1},} \\
& \text { I.S. }(S)^{-1}=I, \quad \text { TS.S. }(T)^{-1}=T S^{2} T \text {, } \\
& \text { T.S. }(T S)^{-1}=I, \quad \text { TR.S. }(T S R)^{-1}=I \text {, } \\
& \text { S.S. }(I)^{-1}=S^{2}, \quad \text { SR.S. }(R)^{-1}=I, \\
& \text { R.S. }(S R)^{-1}=R S R S^{-1}, \quad \text { TSR.S. }(T R)^{-1}=T S R S R T \text {, } \\
& \text { I.R. }(R)^{-1}=I, \quad \text { TS.R. }(T S R)^{-1}=I, \\
& \text { T.R. }(T R)^{-1}=I, \quad \text { TR.R. }(T)^{-1}=I, \\
& \text { S.R. }(S R)^{-1}=I, \quad \text { SR.R. }(S)^{-1}=I, \\
& \text { R.R. }(I)^{-1}=I, \quad \text { TSR.R. }(T S)^{-1}=I,
\end{aligned}
$$

Since $\operatorname{SRTRS}^{-1} T=S T S^{-1} T, \quad \operatorname{TSRTRS}^{-1}=\operatorname{TSTS}^{-1}, \operatorname{TSTS}^{-1}=$ $\left(S T S^{-1} T\right)^{-1}, R S R S^{-1}=S^{-2}$ and $T S R S R T=I$, the generators of $\bar{H}^{2}\left(\lambda_{q}\right)$ are $S^{2}, T S^{2} T$ and $T S T S^{-1}$. Thus $\bar{H}^{2}\left(\lambda_{q}\right)$ has a presentation

$$
\bar{H}^{2}\left(\lambda_{q}\right)=\left\langle S^{2}, T S^{2} T, T S T S^{-1} \mid\left(S^{2}\right)^{q / 2}=\left(T S^{2} T\right)^{q / 2}=\left(T S T S^{-1}\right)^{\infty}=I\right\rangle .
$$

Therefore $\bar{H}^{2}\left(\lambda_{q}\right)$ has the signature $\left(0 ;(q / 2)^{(2)}, \infty^{(2)}\right)$.

Corollary 1. 1) If $q \geq 3$ is an odd integer and if $m$ is a positive even integer such that $(m, q)=1$, then $\bar{H}^{m}\left(\lambda_{q}\right)=\bar{H}^{2}\left(\lambda_{q}\right)$.

2) If $q \geq 3$ is an integer and if $m$ is a positive odd integer, then $\bar{H}^{m}\left(\lambda_{q}\right)=\bar{H}\left(\lambda_{q}\right)$.

Proof. 1) If $q \geq 3$ is an odd integer and if $m$ is a positive even integer such that $(m, q)=1$, then by $(2.1)$, we get

$$
\bar{H}\left(\lambda_{q}\right) / \bar{H}^{m}\left(\lambda_{q}\right) \cong<T, R \mid T^{2}=R^{2}=(T R)^{2}=I>\cong D_{2},
$$

from the relations

$$
R^{2}=R^{m}=I, S^{q}=S^{m}=I \text { and } T^{2}=T^{m}=I .
$$

Since $\bar{H}^{2}\left(\lambda_{q}\right)$ is the only normal subgroup of index 4 , we have $\bar{H}^{m}\left(\lambda_{q}\right)=\bar{H}^{2}\left(\lambda_{q}\right)$.

2) If $q \geq 3$ is an integer and if $m$ is a positive odd integer, then by (2.1), we obtain

$$
\bar{H}^{m}\left(\lambda_{q}\right)=\bar{H}\left(\lambda_{q}\right)
$$

from the relations

$$
R^{2}=R^{m}=I, T^{2}=T^{m}=I \text { and }(R S)^{2}=(R S)^{m}=I .
$$


Theorem 2. Let $q>3$ be an even integer and let $m$ be a positive even integer such that $(m, q)=2$. The normal subgroup $\bar{H}^{m}\left(\lambda_{q}\right)$ is the free product of finite cyclic groups $m$ of order $q / 2$ and the infinite cyclic group $\mathbb{Z}$, i.e.,

$$
\begin{aligned}
& \bar{H}^{m}\left(\lambda_{q}\right)=<\underbrace{(T S)(T S) \ldots(T S)}_{(m-1) \text { times }} T S^{-1}>*<S^{2}>*<T S^{2} T>* \\
& <T S T S^{2} T S^{-1} T>* \ldots *<\underbrace{(T S)(T S) \ldots(T S)}_{(m-2) \text { times }} T S^{2} T \underbrace{\left(S^{-1} T\right)\left(S^{-1} T\right) \ldots\left(S^{-1} T\right)}_{(m-2) \text { times }}>.
\end{aligned}
$$

Proof. By (2.1), we have

$$
\begin{aligned}
\bar{H}\left(\lambda_{q}\right) / \bar{H}^{m}\left(\lambda_{q}\right) & \cong\left\langle T, S, R \mid T^{2}=S^{2}=R^{2}=(T R)^{2}=(R S)^{2}=(T S)^{m}=I\right\rangle, \\
& \cong C_{2} \times D_{m},
\end{aligned}
$$

since $S^{q}=S^{m}=I . \quad$ Now we can choose $\{I, T, S, T S, T S T, T S T S, \ldots$, $\underbrace{(T S)(T S) \ldots(T S)}_{(m-1) \text { times }}, R, T R, S R, T S R, T S T R, T S T S R, \ldots, \underbrace{(T S)(T S) \ldots(T S)}_{(m-1) \text { times }} R\}$ as a Schreier transversal for $\bar{H}^{m}\left(\lambda_{q}\right)$. According to the Reidemeister-Schreier method, we find the generators generators of $\bar{H}^{m}\left(\lambda_{q}\right)$ as

$$
\begin{aligned}
& a_{1}=\underbrace{(T S)(T S) \ldots(T S)}_{(m-1) \text { times }} T S^{-1}, a_{2}=S^{2}, a_{3}=T S^{2} T, a_{4}=T S T S^{2} T S^{-1} T, \ldots, \\
& a_{m+1}=\underbrace{(T S)(T S) \ldots(T S)}_{(m-2) \text { times }} T S^{2} T \underbrace{\left(S^{-1} T\right)\left(S^{-1} T\right) \ldots\left(S^{-1} T\right)}_{(m-2) \text { times }} .
\end{aligned}
$$

Thus $\bar{H}^{m}\left(\lambda_{q}\right)$ has a presentation $\bar{H}^{m}\left(\lambda_{q}\right)=$ $\left\langle a_{1}, a_{2}, a_{3}, a_{4}, \ldots, a_{m+1} \mid\left(a_{2}\right)^{q / 2}=\left(a_{3}\right)^{q / 2}=\left(a_{4}\right)^{q / 2}=\cdots=\left(a_{m+1}\right)^{q / 2}=I\right\rangle$.

Also the signature of $\bar{H}^{m}\left(\lambda_{q}\right)$ is $\left(0 ;(q / 2)^{(m)}, \infty^{(2)}\right)$.

We are only left to consider the case where $(m, 2)=2$ and $(m, q)=d>2$. In this case, the above techniques do not say much about $\bar{H}^{m}\left(\lambda_{q}\right)$. But, we can say something about $\bar{H}^{m}\left(\lambda_{q}\right)$ some special cases of $q$. To do these, we need the following results about the commutator subgroups of $\bar{H}\left(\lambda_{q}\right)$ in [9] and [10] .

Lemma 1. 1) For an odd number $q \geq 3$ :

$$
\bar{H}^{\prime}\left(\lambda_{q}\right)=\left\langle S, T S T \mid S^{q}=(T S T)^{q}=I\right\rangle \cong C_{q} * C_{q} .
$$

2) $\bar{H}^{\prime}\left(\lambda_{q}\right) / \bar{H}^{\prime \prime}\left(\lambda_{q}\right)=<S, T S T \mid S^{q}=(T S T)^{q}=(S . T S T)^{q}=I>\cong C_{q} \times C_{q}$.

3) For an even integer $q>3$ :

$$
\begin{aligned}
\bar{H}^{\prime}\left(\lambda_{q}\right) & =<S^{2}, T S^{2} T, T S T S^{-1} \mid\left(S^{2}\right)^{q / 2}=\left(T S^{2} T\right)^{q / 2}=\left(T S T S^{-1}\right)^{\infty}=I> \\
& \cong C_{q / 2} * C_{q / 2} * \mathbb{Z} .
\end{aligned}
$$


By using Lemma 1 and Theorem 1 we get the following

Corollary 2. Let $q \geq 3$ be an integer. Then $\bar{H}^{\prime}\left(\lambda_{q}\right) \cong \bar{H}^{2}\left(\lambda_{q}\right)$.

Theorem 3. Let $q \geq 3$ be an odd integer and let $m$ be a positive integer. The groups $\bar{H}^{2 q}\left(\lambda_{q}\right)$ are the subgroups of the second commutator subgroup $\bar{H}^{\prime \prime}\left(\lambda_{q}\right)$.

Proof. Since $\bar{H}^{\prime}\left(\lambda_{q}\right)=\bar{H}^{2}\left(\lambda_{q}\right)$, we get $\left(\bar{H}^{2}\left(\lambda_{q}\right)\right)^{q} \leq \bar{H}^{2}\left(\lambda_{q}\right)$ and $\left(\bar{H}^{\prime}\left(\lambda_{q}\right)\right)^{q} \leq$ $\bar{H}^{\prime}\left(\lambda_{q}\right)$. Also we know that

$$
\bar{H}^{\prime}\left(\lambda_{q}\right) /\left(\bar{H}^{\prime}\left(\lambda_{q}\right)\right)^{q}=<S, T S T \mid S^{q}=(T S T)^{q}=(S . T S T)^{q}=\cdots=I>.
$$

Therefore the index $\left|\bar{H}^{\prime}\left(\lambda_{q}\right):\left(\bar{H}^{\prime}\left(\lambda_{q}\right)\right)^{q}\right|$ is greater than or equal to the index $\left|\bar{H}^{\prime}\left(\lambda_{q}\right): \bar{H}^{\prime \prime}\left(\lambda_{q}\right)\right|=q^{2}$. Thus we have

$$
\bar{H}^{2 q}\left(\lambda_{q}\right) \subset \bar{H}^{\prime \prime}\left(\lambda_{q}\right) .
$$

By means of this results, we are going to be able to investigate the subgroups $\bar{H}^{2 q m}\left(\lambda_{q}\right)$. We have by Schreier's theorem the following theorem:

Theorem 4. Let $q \geq 3$ be an odd integer. The groups $\bar{H}^{2 q m}\left(\lambda_{q}\right)$ are free.

Finally, we can only say something the case $q=4$. This Hecke group is very important and studied by many authors, see [1] and [7].

Theorem 5. i) $\left|\bar{H}^{2}\left(\lambda_{4}\right):\left(\bar{H}^{2}\right)^{2}\left(\lambda_{4}\right)\right|=8$.

ii) The group $\left(\bar{H}^{2}\right)^{2}\left(\lambda_{4}\right)$ is a free group of rank 9.

Proof. i) If we take $k_{1}=S^{2}, k_{2}=T S^{2} T$ and $k_{3}=T S T S^{3}$, then the quotient group $\bar{H}^{2}\left(\lambda_{4}\right) /\left(\bar{H}^{2}\right)^{2}\left(\lambda_{4}\right)$ is the group obtained by adding the relation $k_{i}^{2}=I$ to the relations of $\left(\bar{H}^{2}\right)^{2}\left(\lambda_{4}\right)$, for $i \in\{1,2,3\}$. Thus we have

$$
\bar{H}^{2}\left(\lambda_{4}\right) /\left(\bar{H}^{2}\right)^{2}\left(\lambda_{4}\right) \cong C_{2} \times C_{2} \times C_{2} .
$$

Therefore, we obtain $\left|\bar{H}^{2}\left(\lambda_{4}\right):\left(\bar{H}^{2}\right)^{2}\left(\lambda_{4}\right)\right|=8$.

ii) Let $\Sigma=\left\{I, k_{1}, k_{2}, k_{3}, k_{1} k_{2}, k_{1} k_{3}, k_{2} k_{3}, k_{1} k_{2} k_{3}\right\}$ be a Schreier transversal for $\left(\bar{H}^{2}\right)^{2}\left(\lambda_{4}\right)$. Using the Reidemeister-Schreier method, we obtain the generators of $\left(\bar{H}^{2}\right)^{2}\left(\lambda_{4}\right)$ as follows: 


$$
\begin{array}{ll}
I \cdot k_{1} \cdot\left(k_{1}\right)^{-1}=I, & I \cdot k_{2} \cdot\left(k_{2}\right)^{-1}=I, \\
k_{1} \cdot k_{1} \cdot(I)^{-1}=I, & k_{1} \cdot k_{2} \cdot\left(k_{1} k_{2}\right)^{-1}=I, \\
k_{2} \cdot k_{1} \cdot\left(k_{1} k_{2}\right)^{-1}=k_{2} k_{1} k_{2} k_{1}, & k_{2} \cdot k_{2} \cdot(I)^{-1}=I, \\
k_{3} \cdot k_{1} \cdot\left(k_{1} k_{3}\right)^{-1}=k_{3} k_{1} k_{3}^{-1} k_{1}, & k_{3} \cdot k_{2} \cdot\left(k_{2} k_{3}\right)^{-1}=k_{3} k_{2} k_{3}^{-1} k_{2}, \\
k_{1} k_{2} \cdot k_{1} \cdot\left(k_{2}\right)^{-1}=k_{1} k_{2} k_{1} k_{2}, & k_{1} k_{2} \cdot k_{2} \cdot\left(k_{1}\right)^{-1}=I, \\
k_{1} k_{3} \cdot k_{1} \cdot\left(k_{3}\right)^{-1}=k_{1} k_{3} k_{1} k_{3}^{-1}, & k_{1} k_{3} \cdot k_{2} \cdot\left(k_{1} k_{2} k_{3}\right)^{-1}=k_{1} k_{3} k_{2} k_{3}^{-1} k_{2} k_{1}, \\
k_{2} k_{3} \cdot k_{1} \cdot\left(k_{1} k_{2} k_{3}\right)^{-1}=k_{2} k_{3} k_{1} k_{3}^{-1} k_{2} k_{1}, & k_{2} k_{3} \cdot k_{2} \cdot\left(k_{3}\right)^{-1}=k_{2} k_{3} k_{2} k_{3}^{-1}, \\
k_{1} k_{2} k_{3} \cdot k_{1} \cdot\left(k_{2} k_{3}\right)^{-1}=k_{1} k_{2} k_{3} k_{1} k_{3}^{-1} k_{2}, & k_{1} k_{2} k_{3} \cdot k_{2} \cdot\left(k_{1} k_{3}\right)^{-1}=k_{1} k_{2} k_{3} k_{2} k_{3}^{-1} k_{1}, \\
I \cdot k_{3} \cdot\left(k_{3}\right)^{-1}=I, & \\
k_{1} \cdot k_{3} \cdot\left(k_{1} k_{3}\right)^{-1}=I, & \\
k_{2} \cdot k_{3} \cdot\left(k_{2} k_{3}\right)^{-1}=I, & \\
k_{3} \cdot k_{3} \cdot(I)^{-1}=k_{3}^{2}, & \\
k_{1} k_{2} \cdot k_{3} \cdot\left(k_{1} k_{2} k_{3}\right)^{-1}=I, & \\
k_{1} k_{3} \cdot k_{3} \cdot\left(k_{1}\right)^{-1}=k_{1} k_{3}^{2} k_{1}, & \\
k_{2} k_{3} \cdot k_{3} \cdot\left(k_{2}\right)^{-1}=k_{2} k_{3}^{2} k_{2}, & \\
k_{1} k_{2} k_{3} \cdot k_{3} \cdot\left(k_{1} k_{2}\right)^{-1}=k_{1} k_{2} k_{3}^{2} k_{2} k_{1} . &
\end{array}
$$

After some calculations, we get the generators of $\left(\bar{H}^{2}\right)^{2}\left(\lambda_{4}\right)$ as

$$
\begin{array}{lll}
k_{1} k_{2} k_{3} k_{2} k_{3}^{-1} k_{1}, & k_{1} k_{2} k_{1} k_{2}, & k_{3}^{2}, \\
k_{1} k_{2} k_{3} k_{1} k_{3}^{-1} k_{2}, & k_{1} k_{3} k_{1} k_{3}^{-1}, & k_{1} k_{3}^{2} k_{1}, \\
k_{1} k_{2} k_{3}^{2} k_{2} k_{1}, & k_{2} k_{3} k_{2} k_{3}^{-1}, & k_{2} k_{3}^{2} k_{2} .
\end{array}
$$

Also, we find the signature of $\left(\bar{H}^{2}\right)^{2}\left(\lambda_{4}\right)$ as $(1 ; \underbrace{\infty, \infty, \cdots, \infty}_{8 \text { times }})=\left(1 ; \infty^{(8)}\right)$.

Notice that the group $\left(\bar{H}^{2}\right)^{2}\left(\lambda_{4}\right)=\left(H^{2}\right)^{2}\left(\lambda_{4}\right)$ is the principal congruence subgroup $H_{4}\left(\lambda_{4}\right)$ of $H\left(\lambda_{4}\right)$.

Since $\bar{H}^{4 k}\left(\lambda_{4}\right) \leq \bar{H}^{4}\left(\lambda_{4}\right) \leq\left(\bar{H}^{2}\right)^{2}\left(\lambda_{4}\right)$, we are going to be able to investigate the subgroups $\bar{H}^{4 k}\left(\lambda_{4}\right)$. We have by Schreier's theorem the following theorem:

Corollary 3. The groups $\bar{H}^{4 k}\left(\lambda_{4}\right)$ are free.

\section{REFERENCES}

[1] R. Abe and I. R. Aitchison, "Geometry and Markoff'sspectrum for $\mathbb{Q}(i)$," I. Trans. Amer. Math. Soc., vol. 365, no. no. 11, pp. 6065-6102, 2013.

[2] I. N. Cangül, R. Sahin, S. Ikikardes, and O. Koruoğlu, "Power subgroups of some Hecke groups. II." Houston J. Math., vol. 33, no. no. 1, pp. 33-42, 2007.

[3] I. N. Cangül and D. Singerman, "Normal subgroups of Hecke groups and regular maps," Math. Proc. Camb. Phil. Soc., vol. 123, pp. 59-74, 1998.

[4] E. Hecke, "Über die bestimmung dirichletscher reichen durch ihre funktionalgleichungen," Math. Ann., vol. 112, pp. 664-699, 1936.

[5] S. Ikikardes, O. Koruoglu, and R. Sahin, "Power subgroups groups of some Hecke groups," Rocky Mountain Journal of Mathematics, no. No. 2, 2006. 
[6] S. Ikikardes, R. Sahin, and I. N. Cangul, "Principal congruence subgroups of the Hecke groups and related results," Bull. Braz. Math. Soc. (N.S.), vol. 40, no. No. 4, pp. 479-494, 2009.

[7] M. L. Lang, "Normalizers of the congruence subgroups of the Hecke groups $G_{4}$ and $G_{6}$," $J$. Number Theory, vol. 90, no. no. 1, pp. 31-43, 2001.

[8] W. Magnus, A. Karrass, and D. Solitar, Combinatorial Group Theory. New York: Dover Publications, 1976.

[9] R. Sahin and O. Bizim, "Some subgroups of the extended Hecke groups $\bar{H}\left(\lambda_{q}\right)$," Acta Math. Sci., Ser. B, Engl. Ed., vol. 23, no. No.4, pp. 497-502, 2003.

[10] R. Sahin, O. Bizim, and I. N. Cangul, "Commutator subgroups of the extended Hecke groups $\bar{H}\left(\lambda_{q}\right)$," Czechoslovak Math. J., vol. 54(129), no. no. 1, pp. 253-259, 2004.

[11] R. Sahin, S. Ikikardes, and O. Koruoğlu, "On the power subgroups of the extended modular group $\bar{\Gamma}$," Tr. J. of Math., vol. 29, pp. 143-151, 2004.

[12] R. Sahin, S. Ikikardes, and O. Koruoğlu, "Some normal subgroups of the extended Hecke groups $\bar{H}\left(\lambda_{p}\right)$, , Rocky Mountain J. Math., vol. 36, no. no. 3, pp. 1033-1048, 2006.

[13] R. Sahin, O. Koruoğlu, and S. Ikikardes, "On the extended Hecke groups $\bar{H}\left(\lambda_{5}\right)$," Algebra Colloq., vol. 13, no. no. 1, pp. 17-23, 2006.

[14] D. Singerman, "Subgroups of Fuschian groups and finite permutation groups," Bull. London Math. Soc., vol. 2, no. 319-323, 1970.

Authors' addresses

Zehra Sarıgedik

Celal Bayar Üniversitesi, Köprübasi Meslek Yüksek Okulu 45930 Manisa, Turkey

E-mail address: zehra.sarigedik@cbu.edu.tr

Sebahattin İkikardes

Balıkesir Üniversitesi, Fen-Edebiyat Fakültesi, Matematik Bölümü, 10145 Balıkesir, Turkey

E-mail address: skardes@balikesir.edu.tr

\section{Recep Sahin}

Balıkesir Üniversitesi, Fen-Edebiyat Fakültesi, Matematik Bölümü, 10145 Balıkesir, Turkey

E-mail address: rsahin@balikesir.edu.tr 